\title{
Evolution of Entertainment in Computer Games
}

\author{
Zahid Halim \\ FAST-National University of \\ Computer and Emerging \\ Science \\ Islamabad, Pakistan.
}

\author{
M. Fazal-ur-Rehman \\ National University of \\ Computer and Emerging \\ Science \\ Islamabad, Pakistan.
}

\author{
A. Rauf Baig \\ National University of \\ Computer and Emerging \\ Science \\ Islamabad, Pakistan.
}

\begin{abstract}
Games have always been part of human culture since a long time; with the advent of modern day computers there has been a change in trend of human focus towards computer based games. Computer games have seen many evolutions from console based games to modern 3D graphic systems. The question, "which era of games or which version of computer games has been more entertaining?" from the user's point of view, remains of interest to game developers and researchers. As the entertainment that a game carries is of subjective nature and vary from user to user. In this work we propose set of criteria to measure the entertainment value of game. Based on the proposed criteria we compare a set of representative games of two different genres to analyze which era/version of computer games has been more entertaining. For this purpose we first device a set of entertainment metrics which are utilized by computational intelligence techniques to measure and compare games.
\end{abstract}

\section{KEYWORDS}

Measuring Entertainment, Computational Intelligence, Computer Games

\section{INTRODUCTION}

Games have been part of human culture since its inception. People play games for multiple purposes which may include relaxation, time pass and physical activation. With the advent of computers and advancement of different electrical technologies traditional games have been replaced, to a great extent, by computer games and some electrical gadgets based games. These electronics and computer games have been very successful and popular especially in young age groups. Researchers nowadays are interested in how to automatically generate the games themselves or to generate their contents at runtime. All these efforts are to make computer games more attractive and entertaining for human players. There is some work done in the context of automatic generation of game contents and its levels generation listed in the related work section of this paper. Another angle to address the same issue of quantifying entertainment from the point of view of human player will be to somehow compare the entertainment that a game contain for both some old set of games and their newer versions.

In this work we study the evolution of entertainment in computer games using computational intelligence techniques. We take a couple of games from two different genres of games which are board based games and the predator/prey type games for empirical analysis. For the purpose of quantifying/measuring entertainment we define set of metrics these metrics are proposed on the bases of different theories of entertainment in computer games along with different comparable dimensions of game (which include duration, challenge, intelligence and usability); these theories are listed in the related work section of this paper. Once we measure the entertainment value of a game we use this measure for our analysis purpose.

The paper is organized as follows: section 2 lists the related work along with the different theories of entertainment, section 3 covers and explains the games we use for our study, section 4 covers the proposed metrics for measuring entertainment, which are different for each genre of game due to each having different features. Section 5 is dedicated to the controllers we use to automatically play the game and calculate values of entertainment metrics. Section 6 covers the experiments and results and section 7 concludes the paper.

\section{RELATED WORK AND THEORIES OF ENTERTAINMENT}

\section{A. Related Work}

Iida [1], in 2003, has proposed a measure of entertainment for games and used it to analyze the evolution of game of chess over the centuries. This measure is considered to be the pioneer in quantification of entertainment. Even though lida's work is limited to chess variants but the measure of entertainment can be easily applied to other board games. According to this measure, the entertainment value of a game is equal to the length of the game divided by the average number of moves considered by a player on his turn. The game is more entertaining if the value of this measure is low. In [2] Retalis uses board games for elearning. He proposes an e-learning board game that adopts the basic elements of a racing board game but cultivate in students skills like creativity, problem-solving, and imagination, as students are trying to reach the end by improving their performance in a variety of learning activities. In the work done in [2] the issues of measuring entertainment value of the games and its automatic generation of game contents are not addressed. 
Togelius [3] has presented an approach to evolve entertaining car racing tracks for a video game. Tracks were represented as b-splines and the fitness of a track depended on how an evolved neural network based controller (modeled after a player) performed on the track. The objectives were for the car to have made maximum progress in a limited number of time steps (high average speed), high maximum speed (so that at least one section of the track is such that high speeds can be achieved), and high variability in performance (as measured by the final progression made) between trials (so that the track is challenging: neither too easy nor too hard). The game model used for experimentations in [3] is simple both graphically and physically (being 2D).

In [4] three metrics (which are combined into one) have been proposed for measuring the entertainment value of predator/prey games. The first metric is called appropriate level of challenge (T). It is calculated as the difference between the maximum of a player's lifetime and his average lifetime over $\mathrm{N}$ games. This metric has a higher value if the game is neither too hard nor too easy and the opponents are able to kill the player in some of the games but not always. The second metric is behaviour diversity metric (S). It is standard deviation of a player's lifetime over $\mathrm{N}$ games. It has a high value if there is diversity in opponent's behaviour. The third metric is spatial diversity metric $\mathrm{E}\{\mathrm{Hn}\}$. It is the average entropy of grid-cell visits by the opponents over $\mathrm{N}$ games. Its value is high if the opponents move all the time and cover the cells uniformly. This movement portrays aggressive opponent behaviour and gives an impression of intelligent game play. The three metrics are combined into one single metric $\mathrm{I}=[\gamma \mathrm{T}+\delta \mathrm{S}+$ $\varepsilon \mathrm{E}\{\mathrm{Hn}\}] /[\gamma+\delta+\varepsilon]$ where $\mathrm{I}$ is the interest value of the predator/prey game; $\gamma, \delta$ and $\varepsilon$ are weight parameters. The work in [5] is some sort of extension of [4].

In[6], the authors have developed a computer game called "Glove" with three levels of incongruity: hard, easy and balanced. There assumption is that the player would get frustrated or bored respectively, with the first two settings and would enjoy with the third one. In [7] a methodology for optimizing player satisfaction in games on a physical interactive platform known as Playware is demonstrated. An ANN is used to map individual playing characteristics to suggest entertainment preferences for game players. Based on the preferences controllable game parameters are adjusted in real-time in order to improve the entertainment value of the game for the player. Performance of the mechanism is evaluated using a survey. A study of capturing the relation of physiology, beyond heart rate recording, to expressed preferences of entertainment in children's physical game play is presented [8]. A survey experiment raises the difficulties of isolating elements derived from heart rate recordings attributed to reported entertainment and a control experiment for surmounting those difficulties is anticipated. A survey experiment on a larger scale is formulated where more physiological signals like blood volume pulse and skin conductance, are collected and analyzed. Given effective data collection a set of numerical features is extracted from the child's physiological state. The results in [9] indicate that there appears to be increased mental and/or emotional effort in preferred games of children.

\section{B. Entertainment theories:}

There are many theories on entertainment in computer games. According to Csikszentmihalyi's theory of flow $[10,11]$ the optimal experience for a person is when he is in a state of flow. In this state the person is fully concentrated on the task that he is performing and has a sense of full control $[12,13]$. The state of flow can only be reached if the task is neither too easy nor too hard. In other words the task should pose the right amount of challenge. In addition to the right amount of challenge, Malone [14] proposes two more factors that make games engaging: fantasy and curiosity. If a game has the capability of evoking the player's fantasy and makes him feel that he is somewhere else or doing something exotic then that game is more enjoyable than a game which does not do so. Curiosity refers to the game environment. The game environment should have the right amount of informational complexity: novel but not incomprehensible[15].

Koster's theory of fun [16] states that the main source of enjoyment while playing a game is the act of mastering it. If a game is such that it is mastered easily and the player does not learn anything new while playing then the enjoyment value of that game is low. Rauterberg $[16,17]$ has introduced the concept of incongruity as a measure of interest in a task. Given a task, humans make an internal mental model about its complexity. Incongruity refers to the difference between the actual complexity of the task and the mental model of that complexity that a person has of that task. We have positive congruity if this difference is positive and negative congruity otherwise. In case of negative incongruity a person would be able to accomplish the task easily. Interest in a task is highest when the incongruity is neither too positive nor negative. In case of large positive incongruity the humans have a tendency to avoid the task and in situations of large negative incongruity they get bored. This requirement of right amount of incongruity is similar to the right amount of challenge in the concept of flow mentioned above. It has been further proposed that in case of reasonable positive incongruity the humans have a tendency to learn more about the task so that their mental model comes at par with the actual complexity of the task.

\section{GAMES IN THIS WORK}

The games we have selected are in total six, three from the predator/prey genre of game (one new and two old) and three for the board based games. The older version of both genres of games are the most popular games whereas the newer versions are produced using the genetic algorithm and guided by the entertainment metrics used as the fitness function. The older version of the predator/prey game include the game of PacMan and Dead End, whereas for the board based games these are the popular game of chess and checkers. The rules of the newer versions of the game are listed in figure 1 and 2 respectively. 


\begin{tabular}{|c|c|c|c|c|c|c|c|c|c|c|c|c|c|c|}
\hline \multicolumn{3}{|c|}{ Number of predators } & \multicolumn{3}{|c|}{ Movement logic } & \multicolumn{9}{|c|}{ Collision logic } \\
\hline$\underset{\mathscr{D}}{\mathscr{\nu}}$ & $\begin{array}{l}\bar{D} \\
\stackrel{\circlearrowright}{0}\end{array}$ & $\stackrel{\mathscr{D}}{\bar{n}}$ & $\begin{array}{l}\vec{D} \\
\mathscr{\mathcal { L }}\end{array}$ & 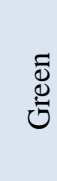 & $\stackrel{\mathscr{E}}{\bar{n}}$ & 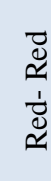 & 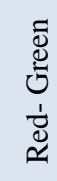 & $\frac{\stackrel{0}{\Xi}}{\frac{1}{0}}$ & 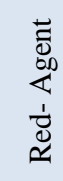 & 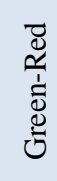 & 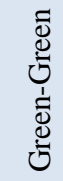 & $\frac{\stackrel{0}{\Xi}}{\frac{1}{0}}$ & 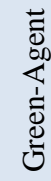 & 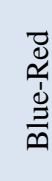 \\
\hline 5 & 15 & 2 & 0 & 3 & 4 & 0 & 1 & 0 & 0 & 0 & 0 & 2 & 1 & 1 \\
\hline \multicolumn{6}{|c|}{ Collision logic } & \multicolumn{9}{|c|}{ Score logic } \\
\hline 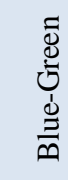 & 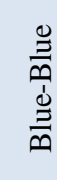 & 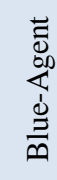 & 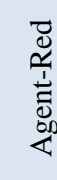 & 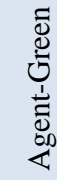 & 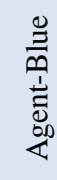 & 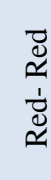 & 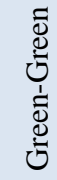 & $\frac{\stackrel{\vartheta}{\Xi}}{\stackrel{\oplus}{\Xi}}$ & 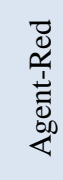 & 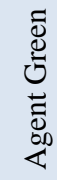 & 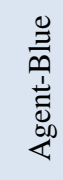 & 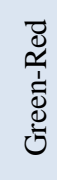 & 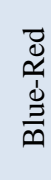 & 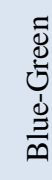 \\
\hline 0 & 0 & 1 & 1 & 0 & 2 & 0 & 0 & 0 & 0 & 1 & 1 & 0 & 0 & 0 \\
\hline
\end{tabular}

Figure 1: Game rules of newer version of predator/prey game.

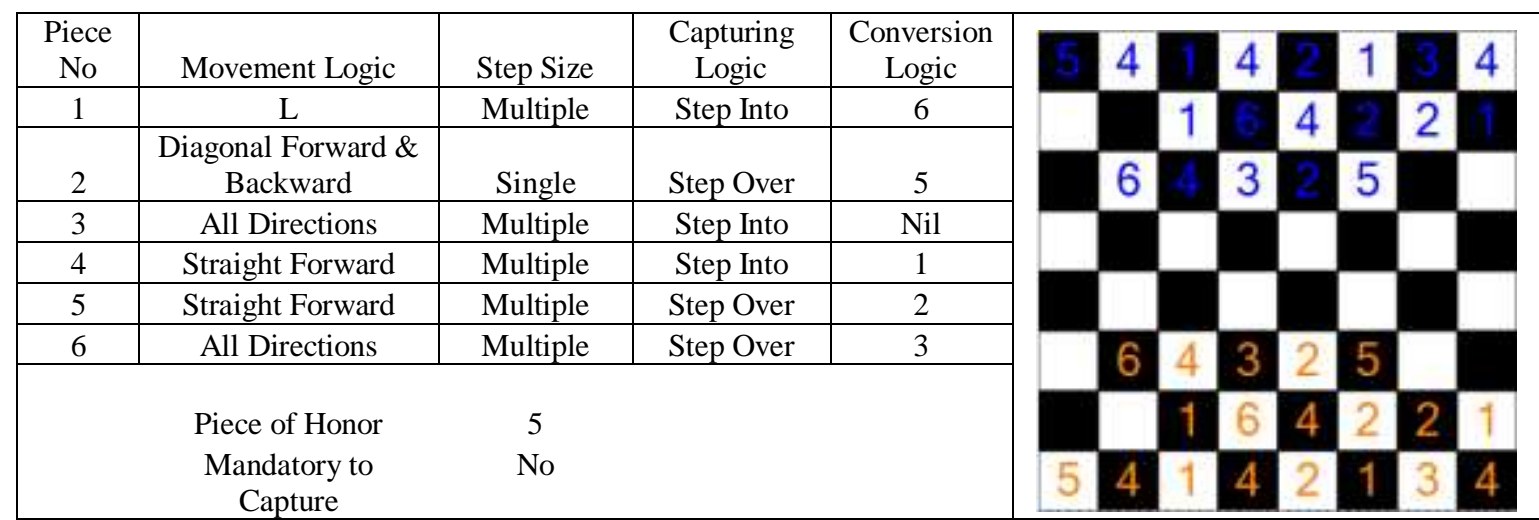

Figure 2: Game rules of newer version of board based game.

The game rules in figure 1 are represented by a chromosome. The rule/feature of the game they represent and the allele are as follows: (1) number of red type predators (2) number of green type predators (3) number of blue type predators (4) movement logic of red type predators (5) movement logic of green type predators (6) movement logic of blue type predators (7) collision logic of red with red (8) collision logic of red with green (9) collision logic of red with blue (10) collision logic of red with agent (11) collision logic of green with red (12) collision logic of green with green (13) collision logic of green with blue (14) collision logic of green with agent (15) collision logic of blue with red (16) collision logic of blue with green (17) collision logic of blue with blue (18) collision logic of blue with agent (19) collision logic of agent with red (20) collision logic of agent with green (21) collision logic of agent with blue (22) score logic of red with red (23) score logic of green with green (24) score logic of blue with blue (25) score logic of agent with red (26) score logic of agent with green (27) score logic of agent with blue (28) score logic of green with red (29) score logic of blue with red (30) score logic of green with blue. The allele for gene 1-3 is $0-20$, for gene 4-6 its 0-4 where they represent still, clockwise, counter-clockwise, random short and random long movement respectively. For gene 7-21 allele is $0-2$ where 0 means nothing happens against collision, 1 means the predator or prey will die and 2 means the predator or pray will be moved to a new randomly chosen location. Allele for gene 22-30 is between -1 to +1 .

The game rules of figure two are simple representing movement logic, step size, capturing and conversion logic of each type of piece. The rules are taken as a common of the games of chess and checkers.

\section{PROPOSED MEASURES}

In this work we are addressing two different genres of games which are board based games and video games (predator/prey games to be specific). Both these genres have different game representation schemes number and types of entities and many other differences, all this makes it difficult to make a single set of metrics for both. Thus proposed entertainment metric depends upon the specific genre of game being addressed. Here we explain the proposed set of four metrics each for the board based games and predator/prey genre of games. 


\section{A. Metrics for Board based games:}

\section{Duration of the game}

The duration of play (D) of a game is calculated by playing the game $n$ times and taking the average number of moves over these $\mathrm{n}$ games. The average value of $\mathrm{D}$ is taken because if the game is played multiple times with a different strategy each time, (or even by the same strategy which has probabilistic components) then we do not get the same value of D every time. For averaging, the game is played $n=100$ times in our experiments. Equation (1) shows the mathematical representation of $\mathrm{D}$.

$$
D=\frac{\sum_{k=0}^{n} L_{K}}{n} \quad(1)
$$

where $\mathrm{L}_{\mathrm{K}}$ is the life of the game playing agent in game $\mathrm{K}$. In order to reward games neither too short nor too long value of raw value of $\mathrm{D}$ is scaled in range $0-1$. The boundaries for scaled value of $\mathrm{D}$ are shown in figure 3 .

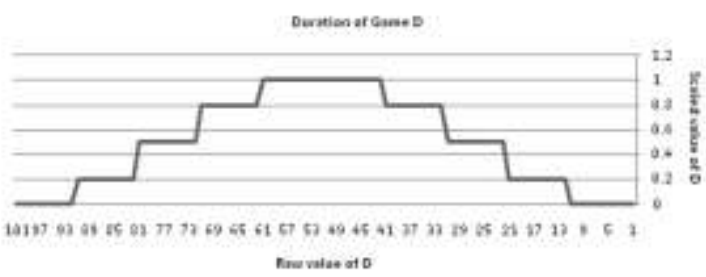

Figure 3: Scaling ranges for raw value for duration of game.

\section{Intelligence for playing the game}

The intelligence (I) is defined as the number of wins of an intelligent controller over a controller making random (but legal) moves. For this purpose the game is played $n$ times ( $n=100$ times in our experiments). Higher number of wins against the random controller means that the game requires intelligence to be played and does not have too many frustrating dead ends. Intelligence $I$ is calculated using equation (2).

$$
I=\frac{\sum_{k=0}^{n} I_{K}}{n}
$$

Where, $I_{K}$ is 1 if intelligent controller wins the game otherwise its 0 .

\section{Dynamism exhibited by the pieces}

This aspect assumes that a game whose rules encourage greater dynamism of movement in its pieces would be more entertaining than a game in which many pieces remain stuck in their cells for the entire duration of the game. The dynamism is captured by the following fitness function given in equation (3).

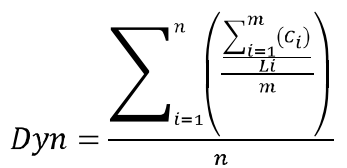

Where,

$\mathrm{C}_{\mathrm{i}}$ is the Number of cell changes made by piece $\mathrm{i}$ during a game

$\mathrm{L}_{\mathrm{i}}$ is life of the piece $\mathrm{i}$

And $\mathrm{m}$ is the total number of pieces in a game.

The dynamism is averaged by calculating it for 100 games. This fitness function has a higher value if the pieces show a more dynamic behaviour.

\section{Usability of the play area}

It is interesting to have the play area maximally utilized during the game. If most of the moving pieces remain in a certain region of the play area then the resulting game may seem strange. The usability is captured using equation (4).

$$
U=\frac{\sum_{i=1}^{n}\left(\frac{\sum_{k=0}^{m}\left(C_{k}\right)}{\left|C_{u}\right|}\right)}{n}
$$

where

$\mathrm{C}_{\mathrm{k}}$ usability counter value for a cell $\mathrm{k}$.

$\left|\mathrm{C}_{\mathrm{u}}\right|$ is the total number of usable cells. $\mathrm{n}$ is 100 as explained previously.

\section{B. Metrics for predator/prey games:}

For the predator/prey genre of games the entertainment metrics include: duration of game, appropriate level of challenge, diversity and usability. The mathematical illustration of these factors may differ if applied to some other genre of games.

\section{Duration of the game}

The duration of play, D, is calculated as in equation 5 .

$$
D=\frac{\sum_{k=0}^{n} L_{K}}{n}
$$

Where $\mathrm{L}_{\mathrm{K}}$ is the life of the game playing agent in game $\mathrm{K}$, And $\mathrm{n}$ is the total number of time the agent plays in this case $\mathrm{n}$ is fixed to 20 .

\section{Appropriate level of challenge}

The challenge $\mathrm{C}$ is converted into a fitness function using equation 6 :

$$
c=e^{\left(\frac{-\left|S_{m}-S_{a}\right|}{S_{m}}\right)}
$$




$$
S_{a}=\frac{\sum_{k=0}^{n} S_{K}}{n} \text { (7) }
$$

$S_{K}$ is score of the agent in game Kth time it plays a game. $\mathrm{n}$ is 100 as explained previously.

Since the value of $S_{a}$ can also be negative, hence we use the following processing:

$$
S_{a}=\left\{\begin{array}{cr}
S_{a}, & \text { if } S_{a} \geq 0 \\
\left|S_{a}\right|+20, & \text { otherwise }
\end{array}\right.
$$

\section{Diversity}

The diversity of the game is based upon the diversity of the pieces in the game.. The diversity is captured by equation 9.

$$
\operatorname{Div}=\frac{\sum_{i=1}^{n}\left(\sum_{k=0}^{m}\left(\partial_{k}\right)\right)}{n}(9)
$$

Where, $\mathrm{m}$ is the total number of pieces (all three types). $\partial_{k}$ Number of cell changes made by piece k during a game. $\mathrm{n}$ is 100 as explained previously.

\section{Usability}

Usability is the fourth and last factor we have considered for our metrics of entertainment. The usability is captured by equation 10 :

$$
U=\frac{\sum_{i=1}^{n}\left(\frac{\sum_{k=0}^{m}\left(c_{k}\right)}{\left|C_{u}\right|}\right)}{n}
$$

where

$\mathrm{C}_{\mathrm{k}}$ usability counter value for a cell $\mathrm{k}$. $\left|\mathrm{C}_{\mathrm{u}}\right|$ is the total number of usable cells. $\mathrm{n}$ is 100 as explained previously.

\section{CONTROLLER}

As the game needs to be played multiple times we need a software based controller for this purpose. For the board based games we need two controllers one making random moves and the other one making moves intelligently whereas for the predator/prey game we need only one rule based controller. Details of each type of controller follow:

\section{A. Random Agent for board games}

As the name suggests the random game playing agent plays the game by randomly selecting a legal move at each step. The agent follows the following algorithm:

Input: Game Board current state

1. Generate all legal moves

2. Store the moves in a queue

3. Shuffle the queue

\author{
4. If Not mandatory to kill \\ 5. Randomly select a move from the queue. \\ 6. Else \\ 7. Select a move that captures an opponent's \\ piece, if such move exists \\ 8. Otherwise, randomly select a move from the \\ queue.
}

Output: Next move to take

The agent initially generates all the legal moves and stores them in a queue. The queue is shuffled once all the moves are saved in it. The shuffling is important, as we take an average of 100 games to calculate the individual metrics values, if the queue is not shuffled then each time the games is played it will use the same sequence of moves to play the game and fitness values will remain the same in each iteration of the game play. If the mandatory to capture bit is "on" in a chromosome which is being evaluated then the agent first tries to find a move that will capture an opponent's piece. If no such move is found it randomly selects a move from the queue of moves.

\section{B. Intelligent Agent for board games}

This type of agent is intelligent as compared to the random one. It generates all the possible one ply depth game boards using a min-max algorithm. Each of the resulting game board is evaluated using a rule based evaluation function and the one with the highest evaluation is selected as a next move.

Evaluation function for this type of agent assigns priorities (weights) to piece-type according to whether its disappearance would cause the game to end, flexibility of movement (more directions and multiple step sizes are better), and capturing logic (capturing by moving into opponent's cell is better). Once the priority of a piece is calculated we multiply each piece with its corresponding weight and calculate weighted summation for self and opponent. The board evaluation is the self weighted summation minus opponents weighted summation. The algorithm for the evaluation function is as follows:

$$
\begin{aligned}
& \text { Input: Game Board current state } \\
& \text { 1. For each piece } \\
& \text { 2. } \quad \text { priority }=0 \\
& \text { 3. For each piece } \\
& \text { 4. if is piece of honor } \\
& \text { 5. } \quad \text { priority }=\text { priority }+1000 \\
& \text { 6. if movement logic all directions } \\
& \text { 7. priority }=\text { priority }+8 \\
& \text { 8. if movement logic diagonal Forward and }
\end{aligned}
$$


17. $\quad$ priority $=$ priority +5

18. if capturing logic step into

19. $\quad$ priority $=$ priority +4

20. if capturing logic step over

21. $\quad$ priority $=$ priority +3

22. Count the number of pieces of Player $A$

23. Multiply the number of pieces of a type with its relevant priority

24. Count the number of pieces of Player $B$

25. Multiply the number of pieces of a type with its relevant priority

26. Calculate boardValue $=$ WeightSumofAWeightSumofB

27. Check if the Piece of Honour is dead add -1000 to boardValue

28. Check if the Piece of Honour is NOT dead add +1000 to boardValue

Output: boardValue

Since we are using mini-max of single ply hence we had to incorporate a mechanism in the evaluation function to overcome the randomness effect near the end of the game when pieces are few and may be far apart. In such case the evaluation function gives same evaluation for all the board positions thus increasing the duration of game. To avoid such situation we restrict the agent to select the move which decreases distance between its own piece and one of an opponent's pieces, provided all next game board position have equal evaluation.

\section{Rule based controller for predator/prey game:}

Rule based controller is implemented as a human supplied rule set. The same controller is used for playing all games (chromosomes) during the entire evolutionary process. Our rule based agent controller is composed of rules formulated to implement the following policy.

According to the game rules, at each simulation step the agent must take exactly one step. The agent looks up, down, left and right. It notes the nearest piece (if any) in each of the four directions, and then it simply moves one step towards the nearest score increasing piece. If there are no score increasing piece present it determines its step according to the following priority list:

- Move in the direction which is completely empty (there is only the wall at the end). If more than one directions are empty move towards the farthest wall (in the hope that subsequent position changes would show it a score increasing piece)

- Move in the direction which contains a score neutral piece. The farthest, the better.

- Move in the direction which contains a score decreasing piece. The farthest, the better.

- Move in the direction which contains a death causing piece. The farthest, the better.

Going into walls is not allowed, and if there is a wall present in the adjoining cell, the possibility of going in that direction is automatically curtailed. The above mentioned controller rules encourage the agent to maximize its score by trying to collide with the piece which increase its score and at the same time try to avoid collision with the rest.

\section{EXPERIMENTS AND RESULTS}

For the purpose of experimentation we calculate the fitness values (defined in section 4) for each genre of games using the new and old version of these games. As there are probabilistic factors involved in each of the game resulting in different fitness values each time a game is played introducing noise. To remove this noise we play each game 100 times using the software controllers for calculating the individual fitness criteria.

For the board based (BB) games we are using two different types of controller table 1-4 list the effect and values of each type of controller on the individual entertainment metrics. The results in table 1-5 suggest that the entertainment value of the popular games of chess, checkers, PacMan and DeadEnd are comparable to those evolved using the proposed entertainment metrics.

\begin{tabular}{|c|c|c|c|c|c|c|c|c|c|}
\hline & \multicolumn{3}{|c|}{$\mathrm{R} \vee \mathrm{R}$} & \multicolumn{3}{|c|}{ R v I } & \multicolumn{3}{|c|}{ I v I } \\
\hline & $\frac{\tilde{d}}{\tilde{U}}$ & 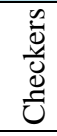 & 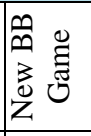 & $\stackrel{\tilde{d}}{\tilde{U}}$ & 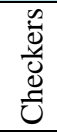 & 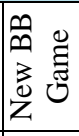 & 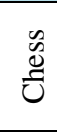 & 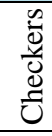 & 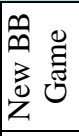 \\
\hline Average & 0.3 & 0.6 & 0.55 & 0.8 & 0.6 & 0.19 & 0.8 & 0.6 & 0.17 \\
\hline Median & 0 & 0.8 & 0.5 & 0.8 & 0.9 & 0.2 & 0.8 & 0.8 & 0 \\
\hline Minimum & 0 & 0 & 0 & 0 & 0 & 0 & 0 & 0 & 0 \\
\hline Maximum & 1 & 1 & 1 & 1 & 1 & 1 & 1 & 1 & 1 \\
\hline Std. Dev & 0.4 & 0.5 & 0.36 & 0.2 & 0.4 & 0.24 & 0.3 & 0.5 & 0.28 \\
\hline
\end{tabular}

Table 1: Duration of Games (data generated by playing100 games) 


\begin{tabular}{|c|c|c|c|c|c|c|c|c|c|}
\hline & \multicolumn{3}{|c|}{$\mathrm{R} \vee \mathrm{R}$} & \multicolumn{3}{|c|}{ R v I } & \multicolumn{3}{|c|}{ I v I } \\
\hline & $\frac{\tilde{d}}{\tilde{U}}$ & 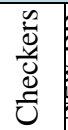 & 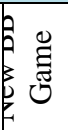 & $\frac{\tilde{d}}{U}$ & 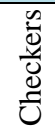 & 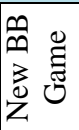 & $\frac{\tilde{d}}{\tilde{U}}$ & $\begin{array}{l}\tilde{\tilde{U}} \\
\frac{\vec{v}}{\tilde{U}} \\
\bar{U}\end{array}$ & 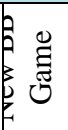 \\
\hline $\begin{array}{l}\text { Wins } \\
\text { Player 1 }\end{array}$ & 53 & 51 & 43 & 0 & 50 & 5 & 45 & 49 & 55 \\
\hline $\begin{array}{l}\text { Wins } \\
\text { Player } 2\end{array}$ & 47 & 48 & 56 & 99 & 50 & 94 & 55 & 46 & 44 \\
\hline Draw & 0 & 1 & 13 & 1 & 0 & 1 & 0 & 5 & 1 \\
\hline Intelligence & - & - & - & 1 & 0.5 & 0.05 & - & - & - \\
\hline
\end{tabular}

Table 2: Intelligence required (data generated by playing100 games)

\begin{tabular}{|c|c|c|c|c|c|c|c|c|c|}
\hline & \multicolumn{3}{|c|}{$\mathrm{R} \vee \mathrm{R}$} & \multicolumn{3}{|c|}{ R v I } & \multicolumn{3}{|c|}{ I v I } \\
\hline & $\frac{\tilde{d}}{\tilde{U}}$ & $\begin{array}{l}\frac{w}{0} \\
\frac{d}{d} \\
\frac{d}{0}\end{array}$ & 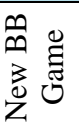 & 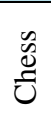 & 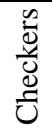 & 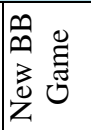 & 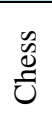 & 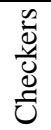 & 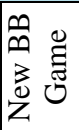 \\
\hline Average & 0.2 & 0.4 & 0.06 & 0.1 & 0.3 & 0.05 & 0.1 & 0.2 & 0.05 \\
\hline Median & 0.1 & 0.3 & 0.06 & 0.1 & 0.2 & 0.05 & 0.1 & 0.2 & 0.05 \\
\hline Minimum & 0.1 & 0.1 & 0.03 & 0.1 & 0.1 & 0.03 & 0.1 & 0.1 & 0.02 \\
\hline Maximum & 1.8 & 3.1 & 0.1 & 0.2 & 0.9 & 0.1 & 0.2 & 0.4 & 0.1 \\
\hline Std. Dev & 0.2 & 0.4 & 0.01 & 0 & 0.2 & 0.01 & 0 & 0.1 & 0.02 \\
\hline
\end{tabular}

Table 3: Dynamism of pieces (data generated by playing100 games)

\begin{tabular}{|c|c|c|c|c|c|c|c|c|c|}
\hline & \multicolumn{3}{|c|}{$\mathrm{R} v \mathrm{R}$} & \multicolumn{3}{|c|}{ R v I } & \multicolumn{3}{|c|}{ I v I } \\
\hline & $\underset{\tilde{d}}{\tilde{U}}$ & $\begin{array}{l}\frac{n}{0} \\
\frac{u}{0} \\
\frac{d}{0}\end{array}$ & $\begin{array}{ll}\infty & 0 \\
\infty & \Xi \\
z & \Xi \\
0 & 0 \\
z & 0\end{array}$ & $\stackrel{\tilde{d}}{\tilde{U}}$ & $\begin{array}{l}\frac{0}{0} \\
\frac{d}{0} \\
\stackrel{0}{0}\end{array}$ & 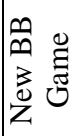 & d্d & 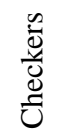 & 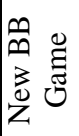 \\
\hline Average & 3.3 & 3.1 & 1.5 & 1.4 & 2.8 & 1.12 & 1.6 & 2.6 & 1.14 \\
\hline Median & 2.4 & 2.6 & 1.44 & 1.4 & 2.6 & 1.09 & 1.6 & 2.6 & 1.11 \\
\hline Minimum & 1.2 & 1.7 & 1 & 1.1 & 1.8 & 1 & 1.1 & 1.8 & 1 \\
\hline Maximum & 11 & 6.2 & 2.27 & 2 & 6.6 & 1.69 & 2.6 & 3.4 & 1.72 \\
\hline Std. Dev & 2.2 & 1.1 & 0.34 & 0.2 & 0.7 & 0.14 & 0.3 & 0.4 & 0.18 \\
\hline
\end{tabular}

Table 4: Usability of Play Area (data generated by playing 100 games)

The individual fitness values for the predator/prey games are listed in table 5 .

\begin{tabular}{|c|c|c|c|c|c|c|c|c|c|c|c|c|c|c|c|}
\hline & \multicolumn{5}{|c|}{ PacMan } & \multicolumn{5}{|c|}{ DeadEnd } & \multicolumn{5}{|c|}{ New PP Game } \\
\hline Matrices & 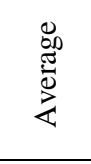 & 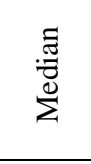 & $\underset{\text { 声 }}{\stackrel{\Xi}{\Xi}}$ & 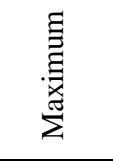 & $\begin{array}{l}\vec{\Xi} \\
\text { Dे } \\
\vec{\Delta}\end{array}$ & 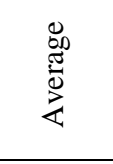 & 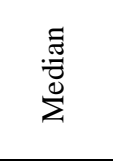 & $\underset{\Xi}{\text { 志 }}$ & 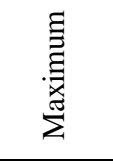 & $\begin{array}{l}\overrightarrow{\tilde{U}} \\
\vec{D} \\
\vec{\omega}\end{array}$ & 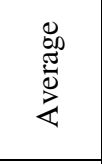 & 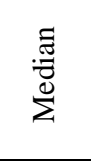 & $\underset{\text { 志 }}{\stackrel{\Xi}{\Xi}}$ & $\begin{array}{l}\text { 志 } \\
\text { 离 } \\
\text { 荥 }\end{array}$ & $\begin{array}{l}\overrightarrow{\tilde{U}} \\
\overrightarrow{\vec{\omega}} \\
\vec{\omega}\end{array}$ \\
\hline $\begin{array}{l}\text { Duration of Game } \\
\text { (D) }\end{array}$ & 48.22 & 34.50 & 8.00 & 164.00 & 35.41 & 76.66 & 80.00 & 29.00 & 80.00 & 9.61 & 56.88 & 48.33 & 15.00 & 105.00 & 20.78 \\
\hline Challenge & 2.03 & 0.99 & 0.24 & 4.55 & 2.33 & 1.04 & 0.88 & 0.33 & 3.32 & 3.21 & 2.33 & 0.57 & 0.45 & 3.68 & 3.40 \\
\hline Diversity & 56.56 & 51.50 & 24.00 & 88.00 & 18.38 & 131.04 & 134.00 & 63.00 & 168.00 & 20.38 & 80.80 & 70.66 & 45.78 & 130.00 & 23.66 \\
\hline Usability & 24.93 & 20.91 & 10.44 & 59.64 & 10.52 & 9.73 & 9.57 & 7.75 & 13.24 & 1.30 & 18.66 & 12.36 & 11.67 & 34.78 & 9.89 \\
\hline
\end{tabular}

Table 5: Individual metrics results for predator/prey games (data generated by playing 100 games) 


\section{CONCLUSION}

The work done in this paper is focused on measuring entertainment based upon a set of entertainment metrics. We address two genres of games which are predator/prey and board based games as they both differ in nature, type and complexity two different set of metrics are proposed. The basic purpose of the work is to enable researchers and game developers to measure and compare the entertainment that a game carries for human users. The proposed entertainment metrics includes duration, challenge, diversity, usability and intelligence as individual components. The results show that the new and popular older versions of each of the addressed genre of game are comparable to each other. For the purpose of find the entertainment value of a game it must be played multiple times. Further work can be done to device a single entertainment metrics of all genres of games. Others directions could be utilizing these metrics in automatic generation of entertaining games or personalized content generation of games based upon players playing patterns.

\section{REFERENCES}

[1] H. Iida, N. Takeshita, and J. Yoshimura, "A Metric for Entertainment of Board Games: Its Application for Evolution of Chess Variants:, In Nakatsu, R., and Hoshino, J., eds., Entertainment Computing: Technologies and Applications (Proceedings of IWEC 2002), pages 65-72. Boston, MA: Kluwer Academic Publishers. 2003

[2] S. Retalis, "Creating Adaptive e-Learning Board Games for School Settings Using the ELG Environment", J. UCS 14(17): 2897-2908,2008

[3] J. Togelius, R. D. Nardi, and S. M. Lucas, "Towards automatic personalised content creation for racing games", in Proceedings of the IEEE Symposium on Computational Intelligence and Games, 2007.

[4] G. N. Yannakakis , John Hallam, ”Towards Optimizing Entertainment In Computer Games, Applied Artificial Intelligence”, v.21 n.10, p.933-971, November 2007.

[5] M. Gallagher and A. Ryan, "Learning to Play Pac-Man: An Evolutionary Rule-based Approach," in Proc. of the 2003 Congress on Evolutionary Computation (CEC'03), 2003, pp. 2462-2469.

[6] G. Lankveld, P. Spronck, M. Rauterberg, "Difficulty Scaling through Incongruity", AIIDE, 2008

[7] K.Compton and M. Mateas, "Procedural Level Design for Platform Games", Proc. 2nd Artificial Intelligence and
Interactive Digital Entertainment Conference (AIIDE '06), Stanford, CA, 2006

[8] G. N. Yannakakis, and J. Hallam, "Entertainment Modeling through Physiology in Physical Play," International Journal of Human-Computer Studies, vol. 66, issue 10, pp. 741-755, October 2008.

[9] R. J. Orr and G. D. Abowd, "The smart floor: a mechanism for natural user identification and tracking", in CHI '00: CHI '00 extended abstracts on Human factors in computing systems, NY, USA: ACM Press, 2000, pp. 275276

[10] M. Csíkszentmihályi, "Flow: The Psychology of Optimal Experience", New York: Harper and Row. ISBN 0-06-092043-2. 1990

[11] M. Csikszentmihalyi and I. Csikszentmihalyi, "Introduction to Part IV in Optimal Experience", Psychological Studies of Flow in Consciousness, Cambridge, UK: Cambridge University Press. 1988.

[12] M. Federoff, "Heuristics and usability guidelines for the creation and evaluation of fun in video games", Ph.D. Dissertation, Indiana University, Bloomington. Unpublished thesis, http://www.melissafederoff.com/thesis.html. 2002

[13] M.Sharma and M. Mehta, M.; Onta ñ'on, S.; and Ram, A. 2007,"Player modeling evaluation for interactive fiction", Technical report, AIIDE 2007 Workshop on Optimizing Player Satisfaction, Palo Alto, California: AAAI Press, 2007

[14] T. W. Malone, “What makes computer games fun?", Byte, vol. 6, pp.258-277, 1981.

[15] D. H. Ackley and M. L. Littman,'Interactions between learning and evolution", In C. G. Langton, C. Taylor, J. D. Farmer, and S. Rasmussen, editors, Artificial Life II, pages 478-507, Reading, MA, 1992. Sante Fe Institute Studies in the Sciences and Complexity, Addison-Wesley.

[16] M. Rauterberg, "Amme: An Automatic Mental Model Evaluation to Analyze User Behavior Traced in a Finite", Discrete State Space, Ergonomics, P-1369-1380. 1993.

[17] M. Rauterberg, "About a Framework for Information and Information Processing of Learning Systems", In Falkenberg, E.; Hesse, W.; and Olibve, A., eds., Information System Concepts, 54-69, IFIP Chapman \& Hall. 1995 